\title{
THE INFRAPATELLAR FAT PAD FROM DISEASED JOINTS INHIBITS CHONDROGENESIS OF MESENCHYMAL STEM CELLS
}

\author{
W. Wei ${ }^{1}$, R. Rudjito ${ }^{1}$, N. Fahy², J.A.N. Verhaar ${ }^{1}$, S. Clockaerts ${ }^{1}$, Y.M. Bastiaansen-Jenniskens ${ }^{1, \S}$ \\ and G.J.V.M. van $\operatorname{Osch}^{1,3, \S, *}$ \\ ${ }^{1}$ Department of Orthopaedics, Erasmus MC University Medical Center, Rotterdam, The Netherlands \\ ${ }^{2}$ Regenerative Medicine Institute, National University of Ireland, Galway, Ireland \\ ${ }^{3}$ Department of Otorhinolaryngology, Erasmus MC University Medical Center, Rotterdam, The Netherlands
}

${ }^{\S}$ Both authors contributed equally

\begin{abstract}
Cartilage repair by bone marrow derived mesenchymal stem cells (MSCs) can be influenced by inflammation in the knee. Next to synovium, the infrapatellar fat pad (IPFP) has been described as a source for inflammatory factors. Here, we investigated whether factors secreted by the IPFP affect chondrogenesis of MSCs and whether this is influenced by different joint pathologies or obesity. Furthermore, we examined the role of IPFP resident macrophages. First, we made conditioned medium from IPFP obtained from osteoarthritic joints, IPFP from traumatically injured joints during anterior cruciate ligament reconstruction, and subcutaneous adipose tissue. Additionally, we made conditioned medium of macrophages isolated from osteoarthritic IPFP and of polarised monocytes from peripheral blood. We evaluated the effect of different types of conditioned medium on MSC chondrogenesis. Conditioned medium from IPFP decreased collagen 2 and aggrecan gene expression as well as thionin and collagen type 2 staining. This antichondrogenic effect was the same for conditioned medium from IPFP of osteoarthritic and traumatically injured joints. Furthermore, IPFP from obese (Body Mass Index >30) donors did not inhibit chondrogenesis more than that of lean (Body Mass Index <25) donors. Finally, conditioned medium from macrophages isolated from IPFP decreased the expression of hyaline cartilage genes, as did peripheral blood monocytes stimulated with pro-inflammatory cytokines. The IPFP and the resident pro-inflammatory macrophages could therefore be targets for therapies to improve MSC-based cartilage repair.
\end{abstract}

Keywords: Cartilage repair, mesenchymal stem cells, infrapatellar fat pad, osteoarthritis, inflammation, obesity.

\footnotetext{
*Address for correspondence:

Gerjo JVM van Osch, PhD

Department of Orthopaedics and Otorhinolaryngology, Ee1655

Erasmus MC University Medical Centre

Wytemaweg 80

3015 CN, Rotterdam, The Netherlands

Telephone Number: + 31-10-7043661

FAX Number: + 31-10-7044690

E-mail: g.vanosch@erasmusmc.nl
}

\section{Introduction}

Articular cartilage has limited self-healing capabilities and surgical intervention using bone marrow stimulation techniques, such as the microfracture procedure, can be used to activate mesenchymal stem cells (MSCs) from the underlying bone marrow to repair the defect (Shapiro et al., 1993; Steadman et al., 2003). However, instead of a normal hyaline cartilage matrix, a fibrocartilaginous cartilage matrix fills up the defect (Frisbie et al., 2003; Kaul et al., 2012; Shapiro et al., 1993). An explanation why hyaline cartilage production is inhibited could be that the cartilage defect is located in a post-traumatic inflammatory environment (Irie et al., 2003). In this post-traumatic inflammatory environment, circulating inflammatory factors stimulate matrix degradation and inhibit chondrogenic differentiation (Heldens et al., 2012).

Besides surgical intervention, research is currently focused on MSC-based tissue-engineered cartilage repair strategies (Hunziker et al., 2015). In these strategies, repair tissues are engineered in-vitro using a combination of MSCs, scaffolds and growth factors and subsequently implanted into the cartilage defect. When implanted, the MSCs in these pre-engineered constructs will be exposed to the same post-traumatic inflammatory environment as the MSCs after the microfracture procedure. An inflammatory environment can inhibit hyaline cartilage matrix production (Wehling et al., 2009).

Another factor that could influence MSC chondrogenic differentiation is obesity. The clinical results after the microfracture procedure are reported to be worse in patients with obesity (Mithoefer et al., 2005). Obesity is known to cause systemic metabolic and inflammatory changes, with increased circulating inflammatory factors (Lumeng and Saltiel, 2011).

Inflammatory factors can be produced by different tissues of the knee joint, the most well described being the synovium and cartilage. The infrapatellar fat pad (IPFP) is an adipose tissue located extra-synovially and intracapsularly. It is richly vascularised, innervated and known to also secrete many adipokines and cytokines (Clockaerts et al., 2012; Clockaerts et al., 2010). Adipose tissue in obese patients has been shown to have an inflammatory phenotype with abundant immune cell infiltration and secretion of inflammatory cytokines (Grant and Dixit, 2015). Furthermore, joint trauma and joint degradation can both increase inflammation in the IPFP and the secretion of inflammatory factors (Bastiaansen-Jenniskens et al., 2012; 
Clockaerts et al., 2012; Gandhi et al., 2011; Gierman et al., 2013).

The IPFP contains immune cells such as macrophages (Bastiaansen-Jenniskens et al., 2012; Klein-Wieringa et al., 2011). Macrophages play an important role in inflammation and wound healing. Macrophages can be categorised into pro-inflammatory macrophages and anti-inflammatory or wound healing macrophages (Mosser and Edwards, 2008; Murray et al., 2014). Inflammation or obesity often results in increased numbers of inflammatory macrophages. Previously, we have shown that only pro-inflammatory and not anti-inflammatory macrophages inhibit MSC chondrogenesis in vitro (Fahy et al., 2014).

Since the IPFP from end-stage OA patients is a source of inflammatory and catabolic cytokines (Clockaerts et al., 2012), it is to be expected that IPFP secreted factors influence other joint tissues. Against our expectation that the factors released from IPFP would stimulate tissue degradation, our previous studies demonstrated that factors released from IPFP from end-stage osteoarthritis (OA) patients inhibit catabolic mediators in cartilage (Bastiaansen-Jenniskens et al., 2012) and stimulate fibrotic processes in synovial fibroblasts (Bastiaansen-Jenniskens et al., 2013). This indicates that the factors released by the IPFP not only have a pro-inflammatory effect on joint environment and but that this effect could be different in each type of joint tissue.

Considering the IPFP's potential to produce factors that influence the joint environment, and the knowledge that the joint environment influences cartilage repair, we hypothesised that factors secreted by the IPFP affect MSC-based cartilage repair. In this study, we investigated whether and how factors secreted by the IPFP affect the chondrogenesis of MSCs in vitro and whether this could be influenced by joint pathology or obesity. Furthermore, we evaluated whether macrophages present in the IPFP could be responsible for the effect seen on MSC chondrogenesis.

\section{Materials and Methods}

\section{Preparation of adipose tissue conditioned medium (CM)}

IPFP tissue was obtained as leftover material from patients with OA who had undergone a total knee replacement (age 66.1 years (53.9-80.2); body mass index (BMI) 29.3 (21.543.7)), or from patients during anterior cruciate ligament (LR) reconstruction (age 29.3 (17.6-48.0); BMI 22.8 (19.3-29.0)), with time from trauma to reconstruction 5.4 months (2-12 months). Subcutaneous adipose tissue (SAT) from patients undergoing total hip replacement was used (age 63.0 (50.2-79.1); BMI 31.3 (24.0-35.8)). Consent was given in accordance with the guidelines of the Federation of Biomedical Scientific Societies (http://www.federa. org) after approval by the local ethical committee (MEC 2012-267). All culture media from now onwards were supplemented with $1.5 \mu \mathrm{g} / \mathrm{mL}$ fungizone and $50 \mu \mathrm{g} / \mathrm{mL}$ gentamicin (both Gibco, Carlsbad, CA, USA). To generate adipose tissue $\mathrm{CM}$, adipose tissue was cut into small pieces of approximately $9 \mathrm{~mm}^{2}$, washed two times with saline and cultured at a concentration of $100 \mathrm{mg} / \mathrm{mL}$ in Dulbecco's
Modified Eagle Medium with Glutamax (DMEM-HG; Gibco) supplemented with $1 \%$ insulin-transferrinselenium (ITS+; BD Biosciences, Erembodegem, Belgium) for $24 \mathrm{~h}$ at $37^{\circ} \mathrm{C}$. Afterwards, the medium was harvested, centrifuged at $250 \times \mathrm{g}$ for $8 \mathrm{~min}$ and the supernatant was stored at $-80{ }^{\circ} \mathrm{C}$ for culture experiments. Unconditioned medium was generated by incubation of DMEM-HG supplemented with ITS + for $24 \mathrm{~h}$ at $37^{\circ} \mathrm{C}$, centrifuged at $250 \times g$ for $8 \mathrm{~min}$ and stored at $-80{ }^{\circ} \mathrm{C}$.

\section{Isolation of macrophages from adipose tissue and preparation of IPFP macrophage CM}

To isolate macrophages from the stromal vascular fraction of adipose tissue, six OA IPFP samples (age 66.6 (54.1-80.2), BMI 28.9 (21.9-33.9)) were used. Adipose tissue samples were cut into pieces of around $9 \mathrm{~mm}^{2}$ and incubated overnight at $37^{\circ} \mathrm{C}$ with $1 \mathrm{mg} / \mathrm{mL}$ collagenase B (Roche, Penzberg, Germany) in DMEM-HG with $10 \%$ FCS. After centrifugation, the supernatant containing the floating adipocytes and fat was removed, and the cell pellet was re-suspended and filtered through a $100 \mu \mathrm{m}$ filter followed by a $40 \mu \mathrm{m}$ filter. The resulting cell suspension was layered on top of $15 \mathrm{~mL}$ Ficoll (Ficoll-Paque ${ }^{\mathrm{TM}}$ PLUS, GE Healthcare, Little Chalfont, UK) and separated by density gradient following centrifugation at $1,000 \times g$ for $15 \mathrm{~min}$. The interphase was removed and washed with phosphate buffered saline (PBS; Gibco) containing $2 \%$ foetal calf serum (FCS) followed by incubation with CD45PE (BD Biosciences) for $1 \mathrm{~h}$ and incubation in $20 \mu \mathrm{L}$ of anti-mouse-IgG magnetic beads (Miltenyi Biotec, Bergisch Gladbach, Germany) per 10,000,000 cells for $30 \mathrm{~min}$ in the dark at $4{ }^{\circ} \mathrm{C}$. CD45 positive cells were separated by magnetic activated cell sorting (MACS, MACS Separation Columns LS and MidiMACS ${ }^{\text {тм }}$ Separator, Miltenyi Biotec) according to manufacturer's instructions. Flow cytometric analysis was performed to confirm a $>90 \%$ CD45+ population purity. The CD45 reduced fraction contained $<10 \% \mathrm{CD} 45+$ cells. Isolated CD $45+$ cells were seeded at a cell density of 100,000 cells $/ \mathrm{cm}^{2}$ in 24 wells plates and cultured overnight at $37{ }^{\circ} \mathrm{C}$ in DMEM-HG supplemented with $10 \%$ FCS. Cells were then carefully washed twice with phosphate-buffered saline (PBS) to remove non-attached cells and DMEM-HG with $1 \%$ ITS+ was added. After $24 \mathrm{~h}$, IPFP CD45+ CM was harvested, centrifuged at $250 \times \mathrm{g}$ for $8 \mathrm{~min}$ and stored at $-80{ }^{\circ} \mathrm{C}$ for further experiments.

\section{Human monocyte isolation, differentiation and CM preparation}

Peripheral blood monocytes were isolated from buffy coats with CD14+ magnetic beads (Miltenyi Biotec) and MACS according to manufacturer's instructions. Monocytes were then seeded at 500,000 cells $/ \mathrm{cm}^{2}$, stimulated to M(LPS+IFN-y) with $100 \mathrm{ng} / \mathrm{mL}$ lipopolysaccharide (LPS; Sigma Aldrich, St. Louis, MO, USA) and $10 \mathrm{ng} / \mathrm{mL}$ interferon-y (IFN-y; PeproTech, Rocky Hill, NJ, USA) or M(IL-4) with $10 \mathrm{ng} / \mathrm{mL}$ interleukin-4 (IL-4; Preprotech) (Murray et al., 2014). Subsequently, M(LPS+IFN-y) and M(IL-4) CM were made as previously described (Fahy et al., 2014; Grotenhuis et al., 2014). Samples of these experiments were previously used in Fahy et al. (2014), 
but additional gene expression analysis were performed for the current study.

\section{Human dermal fibroblast expansion and CM preparation}

Human dermal fibroblast from adult donors (HDFa) were acquired from Gibco (Cat. no. C-013-5C) and were seeded at 5,000 cells $/ \mathrm{cm}^{2}$ in DMEM-HG containing $10 \% \mathrm{FCS}$. After reaching sub-confluency, HDFa were trypsinised and seeded again. For HDFa CM preparation, P4 cells were cultured until subconfluency, washed with PBS three times, and DMEM-HG with ITS+ was added. After $24 \mathrm{~h}$, $\mathrm{CM}$ was harvested, centrifuged at $250 \times \mathrm{g}$ for $8 \mathrm{~min}$ and stored at $-80{ }^{\circ} \mathrm{C}$ for further experiments.

\section{Isolation and culture of human MSCs}

MSCs were isolated from heparinised bone marrow aspirates of patients with OA undergoing total hip replacement ( 9 donors; age 61 years (32-78)). Patients gave informed consent and the study was approved by the local ethical committee of the Erasmus MC, Rotterdam (MEC 2004-142) and Albert Schweitzer Hospital, Dordrecht (MEC 2011-07). Bone marrow aspirates were plated and washed after $24 \mathrm{~h}$ with PBS containing $2 \%$ FCS (Lonza, Verviers, Belgium). MSCs were expanded in Minimum Essential Medium-Alpha ( $\alpha$-MEM; Gibco) supplemented with $10 \% \mathrm{FCS}, 1 \mathrm{ng} / \mathrm{mL}$ fibroblast growth factor 2 (FGF2; R\&D Systems, Minneapolis, MN, USA) and $25 \mu \mathrm{g} / \mathrm{mL}$ ascorbic acid-2-phosphate (Sigma-Aldrich). After reaching sub-confluency, MSCs were trypsinised and seeded again at a density of 2,500 cells $/ \mathrm{cm}^{2}$. MSCs of passage 3 or 4 were used.

\section{Chondrogenic differentiation of MSCs}

MSCs were encapsulated in $1.2 \%$ alginate (CP Kelco, Atlanta, GA, USA) in saline at a density of 4,000,000 cells $/ \mathrm{mL}$. Beads were formed by purging the MSCalginate solution through a $23 \mathrm{G}$ needle and allowing droplets to fall into $102 \mathrm{mM} \mathrm{CaCl}_{2}$ solution. Pellets were made by centrifugation of 200,000 cells in polypropylene tubes. Beads and pellets were cultured with DMEM-HG with ITS + containing $25 \%$ IPFP CM or unconditioned medium. As chondrogenic supplements, we used $0.1 \mu \mathrm{M}$ dexamethasone (Sigma-Aldrich), $10 \mathrm{ng} / \mathrm{mL}$ transforming growth factor- $\beta 1$ (TGF $\beta 1$; R\&D Systems), $25 \mu \mathrm{g} / \mathrm{mL}$ ascorbic acid-2-phosphate (Sigma-Aldrich), $40 \mu \mathrm{g} / \mathrm{ml}$ L-proline (Sigma-Aldrich) and $1 \mathrm{mM}$ sodium pyruvate (Gibco). MSC beads were cultured in $100 \mu \mathrm{L}$ medium per bead and pellets were cultured in $500 \mu \mathrm{L}$ medium per pellet. Medium was refreshed 3 times a week. Beads and pellets were harvested after $28 \mathrm{~d}$.

For the experiments with macrophages, obese versus lean IPFP, osteoarthritic versus post-joint trauma IPFP, and SAT versus IPFP, beads were first cultured in DMEM-HG containing ITS + and chondrogenic supplements for $14 \mathrm{~d}$. From day 14-17, parts of the DMEM-HG with ITS+ was replaced by conditioned medium or an equal percentage of unconditioned medium. For the experiments with IPFP and SAT CM, we replaced $25 \%$ of the DMEM-HG containing ITS + . For the macrophages experiments, we replaced $50 \%$ of the DMEM-HG containing ITS+ with
CD45 CM or $20 \%$ with M(LPS+IFNy)/M(IL-4) CM. For the CD45 experiments, the lowest cell number used to generate $\mathrm{CM}(0.23 \mu \mathrm{g}$ DNA) was set at $50 \%$ and for the $\mathrm{M}(\mathrm{LPS}+\mathrm{IFN \gamma}) / \mathrm{M}(\mathrm{IL}-4)$ experiments, the average cell number used to generate $\mathrm{CM}(1.64 \mu \mathrm{g}$ DNA) was set at $20 \%$. All subsequent donors were corrected accordingly to assure that medium conditioned by an equal number of cells was added. Chondrogenic supplements were always added after mixing the medium with $\mathrm{CM}$ or unconditioned medium. In this way, all conditions had the same final concentration of all supplements. Beads were harvested at day 17. All media were refreshed $24 \mathrm{~h}$ prior to harvest.

\section{Gene expression analysis}

MSC alginate beads were harvested in ice-cold $55 \mathrm{mM}$ sodium citrate and dissolved after gentle agitation at $4{ }^{\circ} \mathrm{C}$. The solution was then centrifuged at $400 \times g$ for 8 min at $4{ }^{\circ} \mathrm{C}$ and cell pellets were re-suspended in $650 \mu \mathrm{LRNA}$-Bee (TelTest, Friendswood, TX, USA) per bead. Chloroform (Sigma-Aldrich) was added to all samples at $200 \mu \mathrm{L} / \mathrm{mL}$ RNA-Bee. RNA was further purified using a RNeasy Micro Kit (Qiagen). RNA concentration and quality was measured using a NanoDrop ND1000 UV-Vis Spectrophotometer (Isogen Life Science, De Meem, The Netherlands). RNA was reverse-transcribed to cDNA using the RevertAid First Strand cDNA Synthesis Kit (Fermentas, Waltham, MA, USA) according to manufacturer's instructions. qRT-PCR was performed in $10 \mu \mathrm{L}$ reactions on cDNA using the CFX96 Touch $^{\mathrm{TM}}$ Real-Time PCR Detection System (Bio-Rad, Hercules, CA, USA). Expression of glyceraldehyde-3-phosphate dehydrogenase (GAPDH), hypoxanthine phosphoribosyltransferase 1 (HPRT1), collagen type II a1 (COL2A1), aggrecan $(A C A N)$, collagen type I a1 $(C O L 1 A 1)$ and versican $(V C A N)$ were determined with qRT-PCR. The genes GAPDH and HPRT were used as housekeepers. Gene expression was analysed according to the $\Delta \Delta \mathrm{Ct}$ method, with the condition treated with unconditioned medium used as control and set at 1.0. Expression levels of other conditions are expressed relative to this control condition. The primer efficiency for $G A P D H$ was 1.06 , for HPRT 0.96 , for COL2A1 1.02, for ACAN 0.95 , for $C O L 1 A 10.97$ and $V C A N 0.95$. The mean $\mathrm{Ct}$ value for GAPDH was 22.9 and for HPRT 28.8. Difference in $\mathrm{Ct}$ values between the samples within each experiment was less than $1 \mathrm{Ct}$ and there were no significant differences between the conditions (data not shown). Gene expression values after normalisation to housekeepers are used to calculate $C O L 2 A 1 / C O L 1 A$ and $A C A N / V C A N$ ratios.

Primer sequences were as follows: $G A P D H$, forward: GTCAACGGATTTGGTCGTATTGGG, reverse: TGCCATGGGTGGAATCATATTGG, and probe Fam: TGGCGCCCCAACCAGCC. HPRT forward: TATGGACAGGACTGAACGTCTTG, reverse: CACACAGAGGGCTACAATGTG, and probe Fam: CGCCCAATACGACCAAATCCGTTGAC. COL2A1 forward: GGCAATAGCAGGTTCACGTACA, reverse: CGATAACAGTCTTGCCCCACTT, and probe Fam: CCGGTATGTTTCGTGCAGCCATCCT. $A C A N$ forward: TCGAGGACAGCGAGGCC, reverse: TCGAGGGTGTAGCGTGTAGAGA, and probe Fam: ATGGAACACGATGCCTTTCACCACGA. COL1A1 
forward: CAGCCGCTTCACCTACAGC, reverse: TTTTGTATTCAATCACTGTCTTGCC, and probe Fam: CCGGTGTGACTCGTGCAGCCATC. VCAN forward: TGGAATGATGTTCCCTGCAA, reverse: AAGGTCTTGGCATTTTCTACAACAG, and probe Fam: CTGGCCGCAAGCAACTGTTCCTTT.

\section{Quantification of DNA and glycosaminoglycan (GAG) content}

Alginate beads were dissolved in sodium citrate and digested with papain. (Sigma Aldrich). Pellets were digested with proteinase K (Sigma Aldrich). DNA was subsequently quantified by spectrophotometric detection of ethidium bromide (Gibco) binding at 340 and $590 \mathrm{~nm}$. GAG content was determined with dimethylmethylene blue (Polysciences, Warrington, PA, USA) spectrophotometrically at $530 \mathrm{~nm}$ and $590 \mathrm{~nm}$ (Enobakhare et al., 1996) and at pH 1.75.

\section{Histological evaluation of MSC chondrogenesis in pellet culture}

$6 \mu \mathrm{m}$ thick paraffin sections were made of the pellets and thionin staining was performed. For immunohistochemical staining, sections were incubated with monoclonal antibody against collagen type 2 (DSHB, Iowa City, IA, USA, \#II-II 6B3) and collagen type 1 (Abcam, Cambridge, UK, \#6308) or IgG1 isotype control (Dako Cytomation, Heverlee, Belgium, \#X0931). Sections were then incubated with Link and Label (Biogenex, Fremount, CA, USA, HK-321-UK) and freshly prepared new-fuchsin was used as substrate.

\section{Evaluation of macrophage subtypes in adipose tissue} $10 \mu \mathrm{m}$ cryosections were made of 13 samples of OA IPFP (age 65.3 (54.3-74.1), BMI 28 (21.5-35.8)), 7 samples of LR IPFP (age 29.9 (21.3-48), BMI 25.6 (19.3-38.5)) and 9 samples of SAT (age 63.1 ( $\pm 50.2-79.1)$, BMI 32.3 (25.947.2)). After fixation in acetone, sections were incubated with monoclonal antibodies against CD68 (Dako, \#EMB11), CD11c (Chemicon, Millipore, Billerica, MA, USA, \#EP1347Y) and CD206 (Abcam, \#64693). Mouse IgG (Dako) were used as isotype controls. Subsequently, sections were incubated with Link and Label (BioGenex) and freshly prepared new-fuchsin was used as substrate. A cell was considered positive when stained red. Samples were ranked based on their relative number of positive cells in three sections per sample by two blinded observers as previously described (Bastiaansen-Jenniskens et al., 2012). Ranking was performed for each marker separately and for each sample, the average rank of the two observers (WW and RR) was used in the analysis.

\section{Statistical analysis}

IBM SPSS statistics 21 (IBM Corporation, Armonk, NY, USA) was used for statistical analysis. The Shapiro-Wilk test was used to test data for normality and the Levene's test for homogeneity of variance. No data were normally distributed. For gene analysis and GAG measurement, if not otherwise stated, the Kruskal-Wallis test was used followed by a post-hoc Bonferroni correction. For immunohistochemical ranking, the Mann-Whitney U test was used. $p<0.05$ was considered statistically significant.

\section{Results}

\section{IPFP negatively impacts chondrogenic differentiation} of MSCs

COL2A1 and $A C A N$ were only detectable after TGF $\beta 1$ was added (Fig. 1). IPFP from OA donors statistically significantly lowered the gene expression of MSCs for COL2A1 $(p<0.001), A C A N(p<0.001)$ and VCAN, without affecting $C O L 1 A 1$ (Fig. 1A). This resulted in a lower COL2A1/COL1A1 $(p<0.001)$ and $A C A N / V C A N$ ratio $(p<0.05)$ (Fig. $1 \mathrm{~A})$. This effect was absent when $\mathrm{CM}$ was heated to $95^{\circ} \mathrm{C}$ for $10 \mathrm{~min}$ before use, indicating that the effects are caused by proteins produced by IPFP (Fig. 1B). GAG content of the alginate beads was increased by IPFP (Fig. 2A). To investigate the effects of IPFP on matrix production further, pellet culture was used. IPFP decreased thionin and immunohistochemical staining of collagen type 2, whereas collagen type 1 staining was not influenced (Fig. 2C). Furthermore, IPFP decreased GAG content of pellets (Fig. 2D and E).

\section{Effect of obese versus lean IPFP and osteoarthritic versus traumatic joint IPFP on MSC chondrogenesis} Obesity increases the release of inflammatory factors by adipose tissue. Therefore, we hypothesised that CM from obese IPFP donors (BMI 33.1 (30-35.8)) would reduce MSC chondrogenesis more than CM from lean IPFP (BMI 23.1 (21.5-24)) donors. However, chondrogenic markers were not more inhibited by $\mathrm{CM}$ from obese than by $\mathrm{CM}$ from lean donors (Fig. 3A).

Next, we compared the effect of CM from LR IPFP (BMI 22.9 (22.0-25.0)) with BMI matched OA IPFP (BMI 23.1 (21.5-24.0)). Chondrogenic gene expression was not statistically significantly different between MSCs exposed to CM from LR IPFP and CM from OA IPFP. Interestingly, LR IPFP CM statistically significantly increased COL1A1 compared to control and OA IPFP CM $(p=0.023)$ (Fig. 3B).

\section{Chondrogenic differentiation of MSCs is more affected by IPFP than by SAT}

To investigate whether the effect of adipose tissue of the joint (IPFP) is different from subcutaneous adipose tissue (SAT), we added CM made from SAT to MSCs during chondrogenic differentiation. SAT statistically significantly lowered gene expression of COL2A1 and $A C A N(p<0.01)$, but did not influence the COL2A1/COL1A1 and ACAN/ $V C A N$ ratio (Fig. 4A). To investigate whether this is due to a difference in the concentration of factors released by IPFP and SAT, we increased the percentage of SAT CM added to culture up to $50 \%$. In contrast to the effect of IPFP, the effect of SAT on chondrogenesis was not dosage dependent (Fig. 4B). 
A

COL2A1

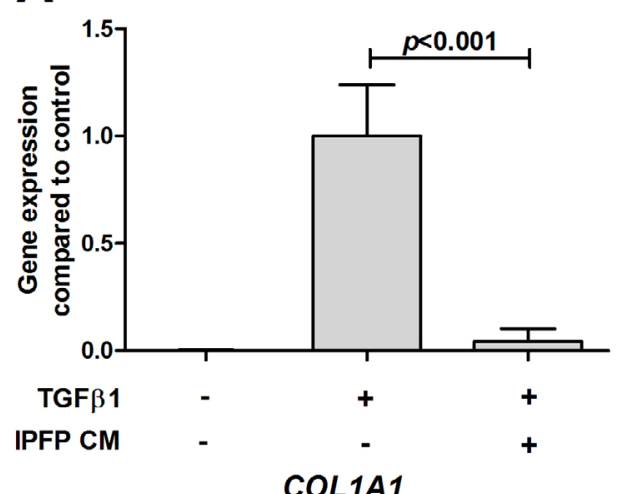

COL1A1

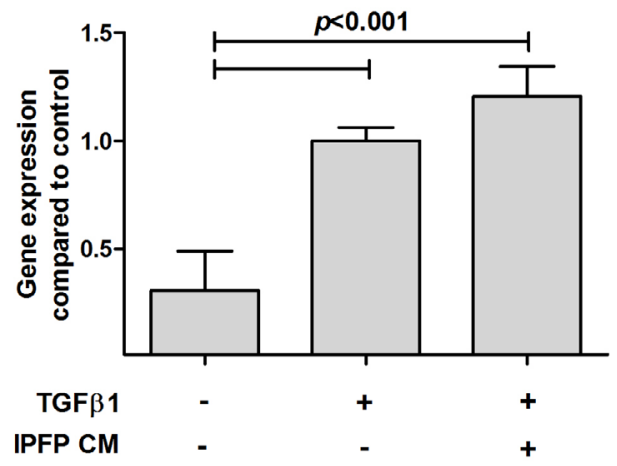

COL2A1 / COL1A1

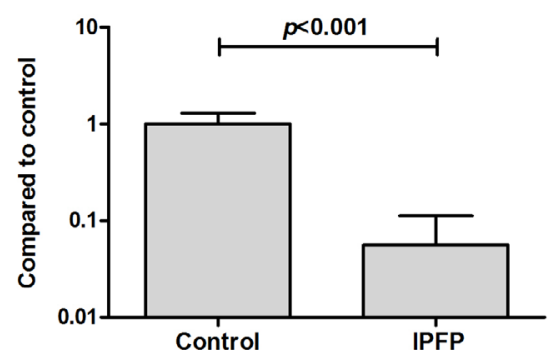

B

COL2A1

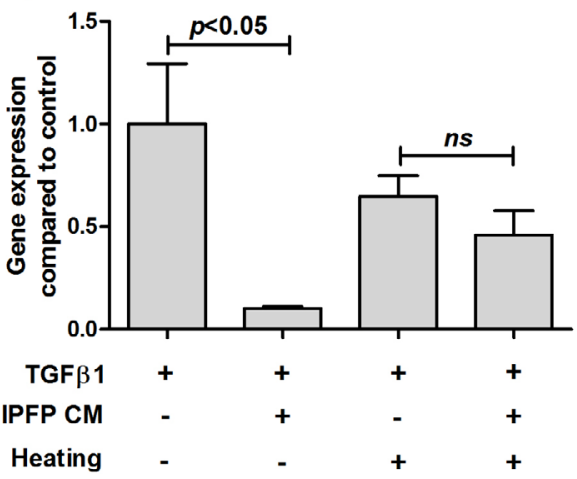

ACAN
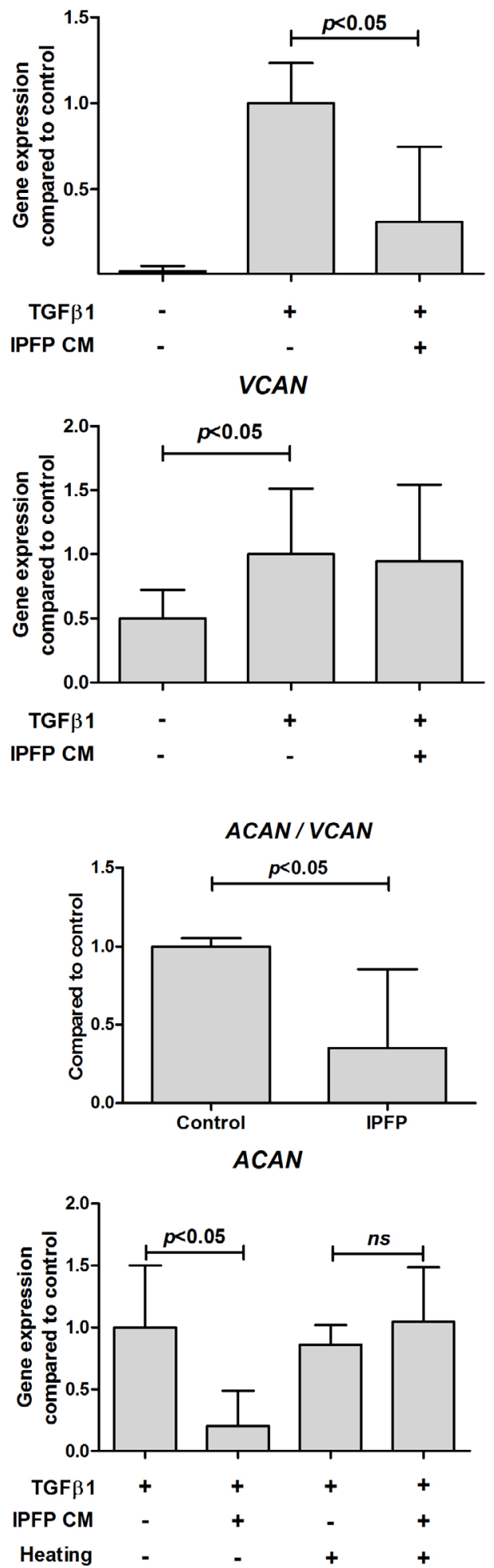

Fig. 1. IPFP inhibits chondrogenic differentiation of MSCs. (A) MSC alginate beads were treated with $25 \%$ IPFP CM for $28 \mathrm{~d}$ of culture in chondrogenic medium with TGF $\beta 1$. The control condition was treated with $25 \%$ unconditioned medium with chondrogenic medium with TGF $\beta 1$. Relative gene expression of COL2A1, ACAN, COL1A1 and VCAN normalised to the control condition and COL2A1/COL1A1 and ACAN/VCAN ratio. CM were pooled from 3-5 different IPFP donors and used to treat 3 MSC donors, with each condition performed in triplicate. (B) MSC alginate beads were treated with $25 \%$ CM from IPFP from day 14-17 of chondrogenic stimulation. CM or unconditioned medium were heated for $10 \mathrm{~min}$ at $95^{\circ} \mathrm{C}$ and allowed to cool down to room temperature before addition to MSC alginate beads. Relative gene expression of COL2A1 and ACAN in MSCs after treatment, normalised to the control condition. One pool of CM from 3 IPFP donors was used to treat 1 MSC donor, with each condition performed in triplicate. The mean $\mathrm{Ct}$ value in the control condition for COL2A1 was 23.2, for ACAN 27.3, for COL1A1 19.7 and for VCAN 24.3. Values represent the mean \pm standard deviation (SD). 

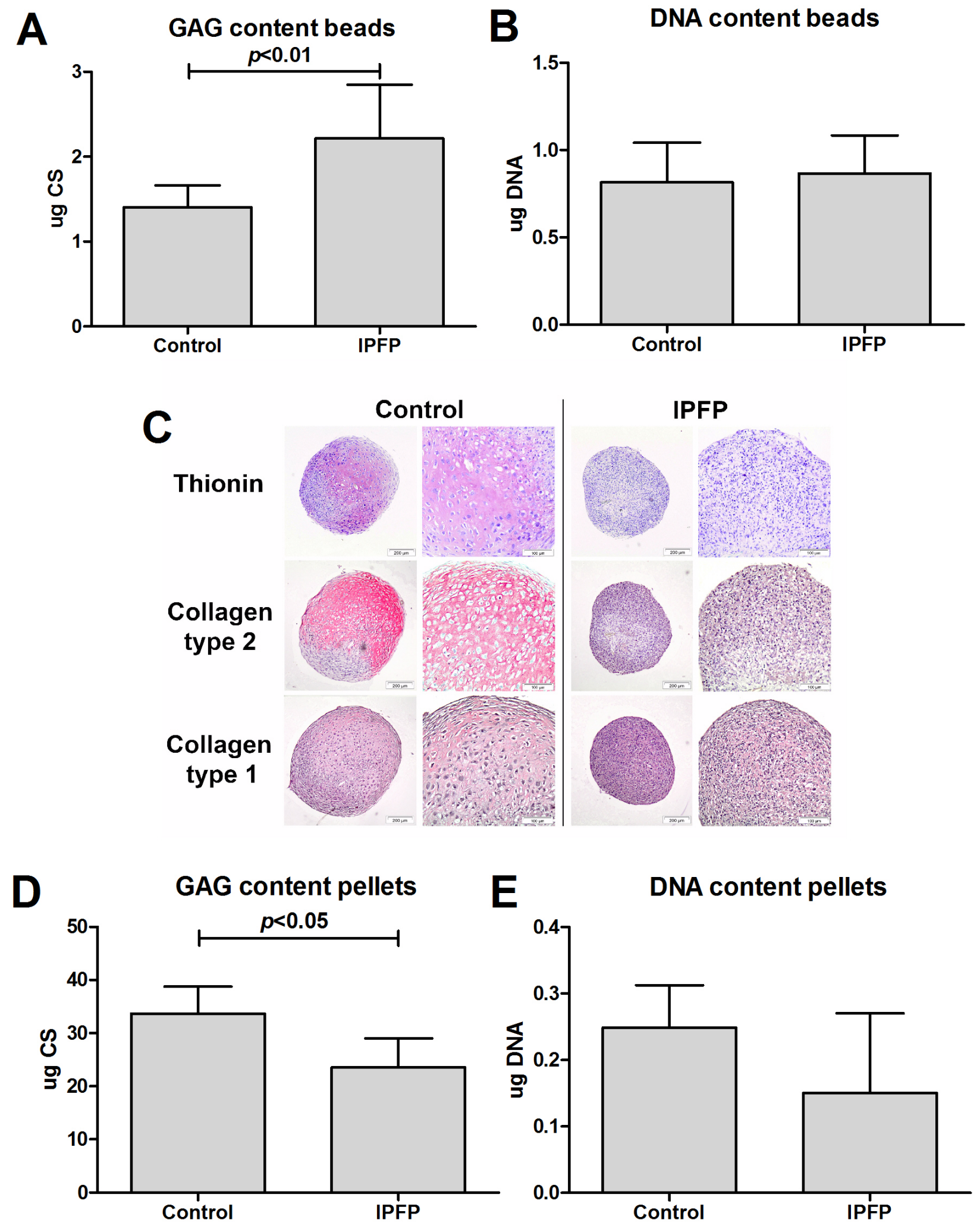

Fig. 2. IPFP inhibits chondrogenic differentiation of MSCs. All conditions were chondrogenically stimulated with TGF $\beta 1$ during the entire culture period. MSC alginate beads and pellets were treated with $25 \%$ IPFP CM whereas the control condition was treated with $25 \%$ unconditioned medium for $28 \mathrm{~d}$. (A) Total GAG content and (B) total DNA content of alginate beads. (C) Representative images of pellets from $1 \mathrm{MSC}$ donor stained for glycosaminoglycans with thionin or collagen type 2 and collagen type 1 using immunohistochemistry. (D) Total GAG content and (E) total DNA content of pellets. Different pools of CM from 3 different IPFP donors were used for each experiment. For total GAG and DNA content in beads (Panels A and B), 3 MSC donors were used with each condition performed in triplicate. For the pellet culture experiment (Panels C-E), 2 MSC donors were used with 5 pellets per condition. Values represent mean $\pm \mathrm{SD}$. The Mann-Whitney U test was used to determine statistical significance. 

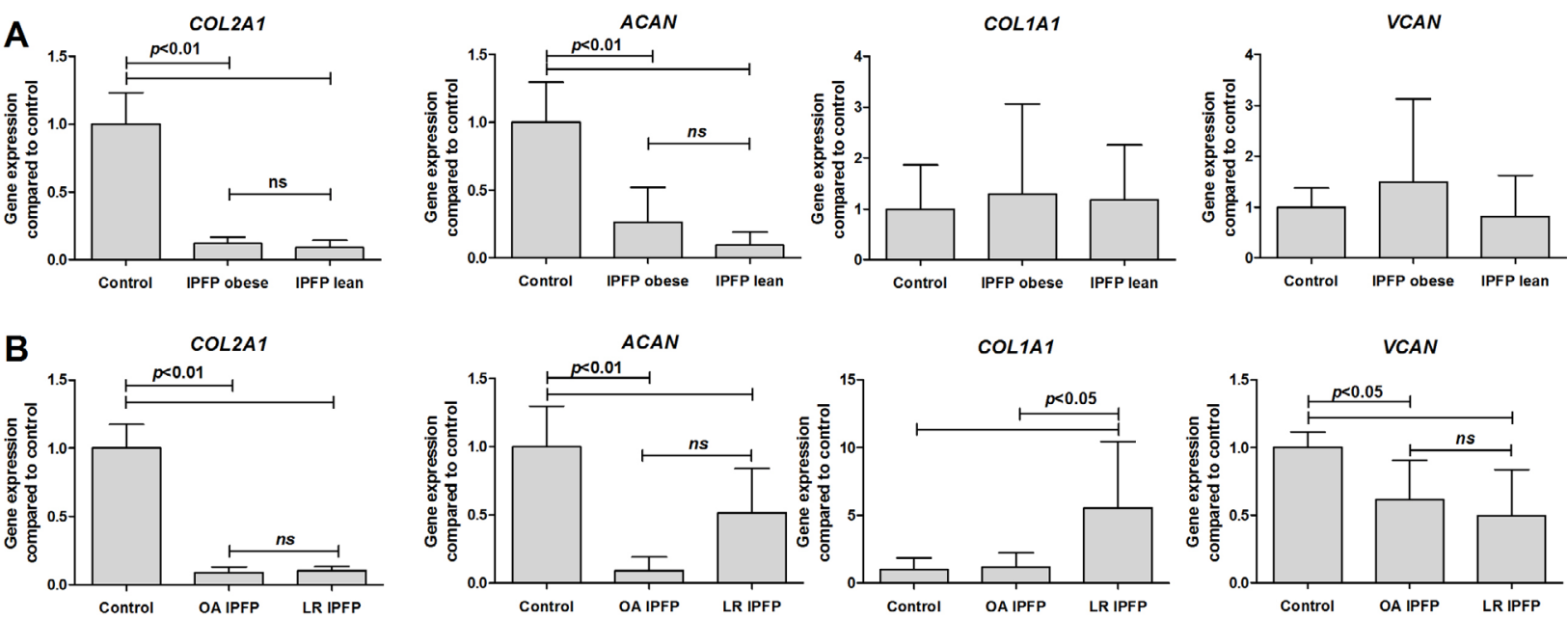

Fig. 3. Effect of obese versus lean IPFP and osteoarthritic versus traumatic joint IPFP on MSC chondrogenesis. All conditions were chondrogenically stimulated with TGF $\beta 1$ during the entire culture period. MSC alginate beads were treated with $25 \%$ IPFP CM from day 14-17. The control condition was treated with $25 \%$ unconditioned medium. Relative gene expression of COL2A1, ACAN, COL1A1 and VCAN in MSCs after treatment with CM from (A) obese $(\mathrm{BMI}>30)$ or lean $(\mathrm{BMI}<25)$ OA IPFP donors, and (B) OA or BMI-matched LR IPFP donors. Gene expression was normalised to the control condition per MSC donor. CM from 6 obese OA IPFP, 6 lean OA IPFP and 6 LR IPFP donors were used. Three different pools of CM were made with each pool a selection of three different IPFP donors. Three MSC donors were used, with each condition performed in triplicate. Values represent the mean $\pm \mathrm{SD}$.
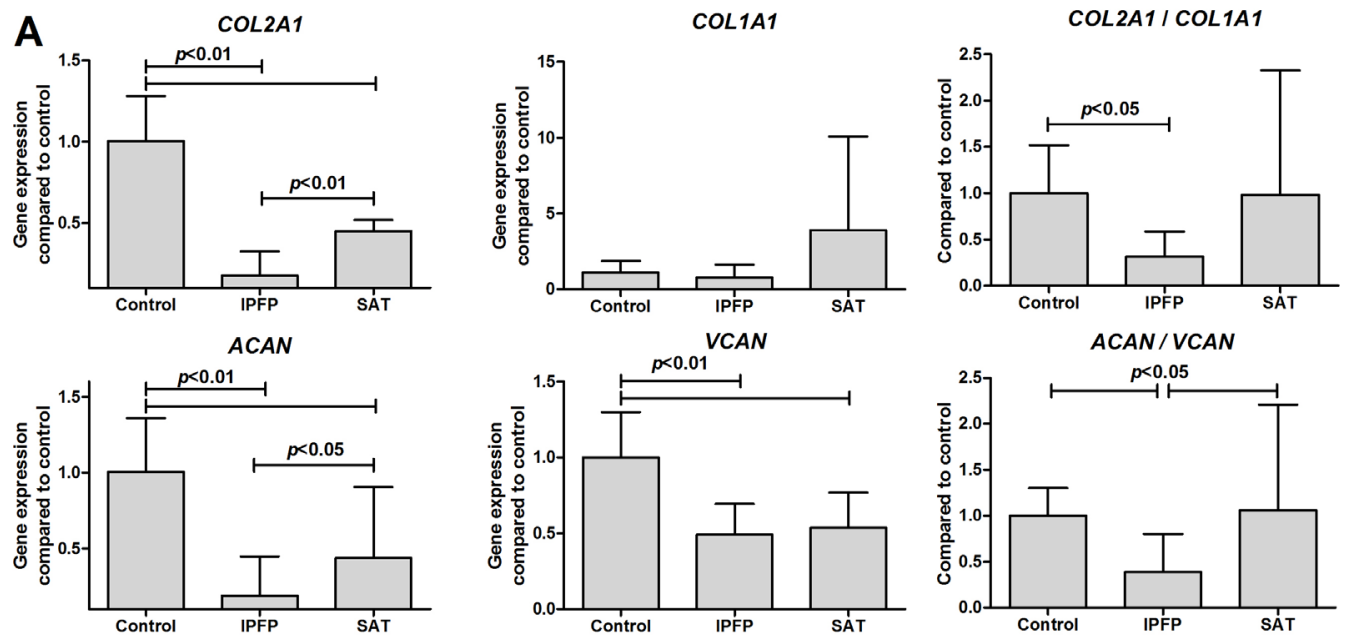

B

COL2A1

ACAN
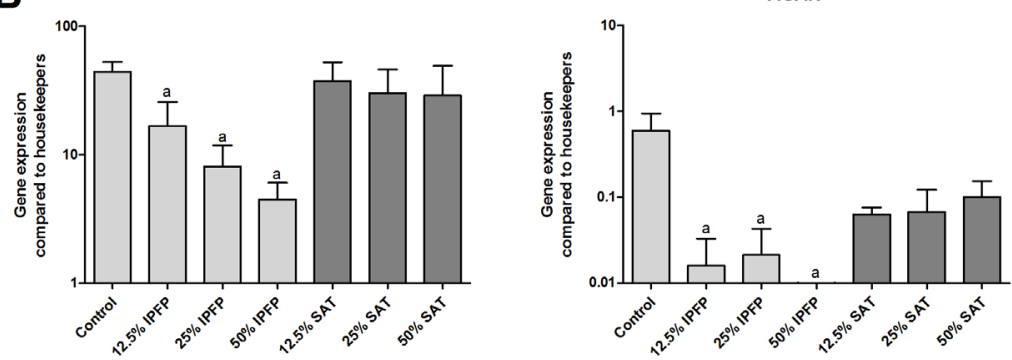

Fig. 4. MSC chondrogenesis is more influenced by IPFP than subcutaneous adipose tissue (SAT). MSC alginate beads were treated with CM from IPFP or SAT from day 14-17 of culture. The control condition was treated with $25 \%$ unconditioned medium. All conditions were chondrogenically stimulated with TGF $\beta 1$ during the entire culture period. (A) $25 \%$ of CM was used and CM were pooled from 3 pools of different 3 IPFP or 3 pools of different 3 SAT donors and used to treat $3 \mathrm{MSC}$ donors. Each experiment was performed in triplicate. Relative gene expression of COL2A1, ACAN, COL1A1, VCAN, COL2A1/COL1A1 ratio and ACAN/VCAN ratio in MSCs after treatment. Gene expression was normalised to the control condition per MSC donor. (B) Different percentages of CM were used and CM were pooled from 3 IPFP or 3 SAT donors and used to treat 1 MSC donor, with each condition performed in triplicate. Relative gene expression of COL2A1 and ACAN compared to housekeeping genes in MSCs after treatment. All conditions received the same final concentration of all chondrogenic supplements. Values represent the mean $\pm \mathrm{SD} .{ }^{\mathrm{a}} p<0.05$ versus control using the Mann-Whitney-U test. 
A

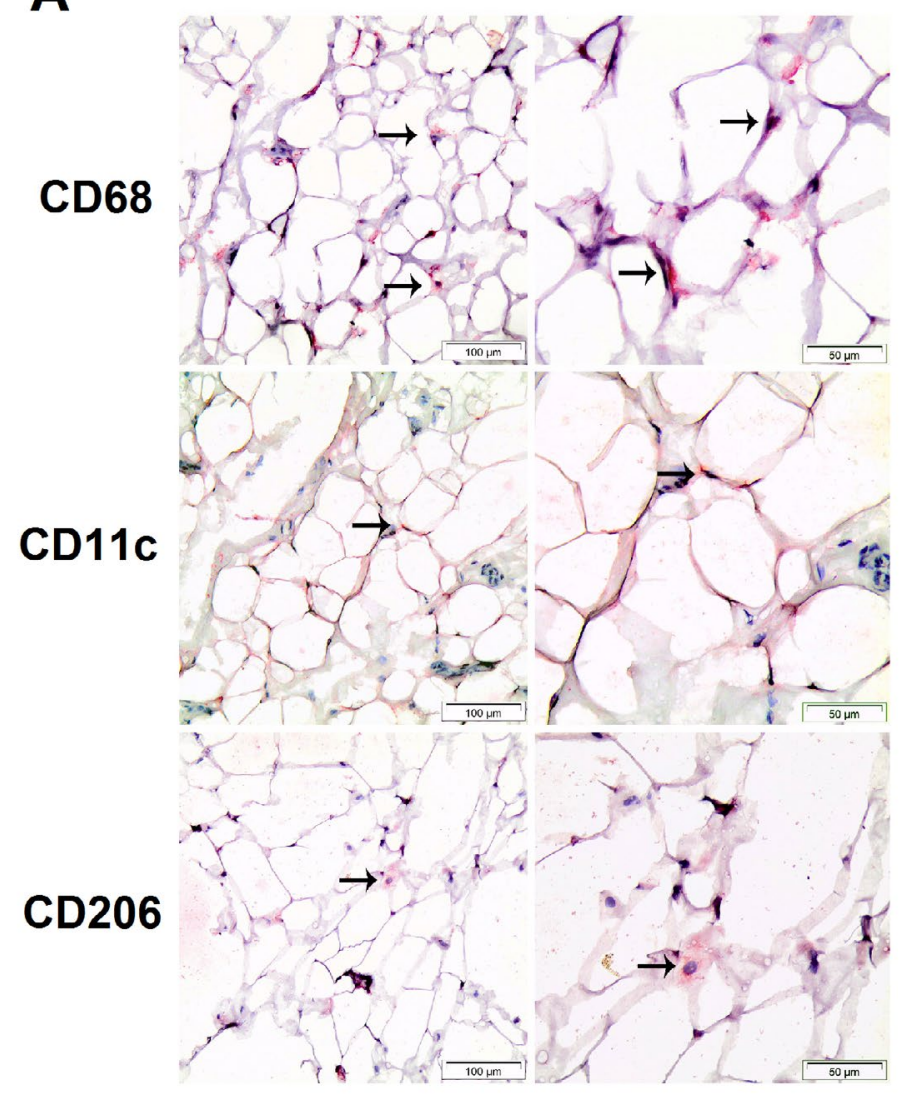

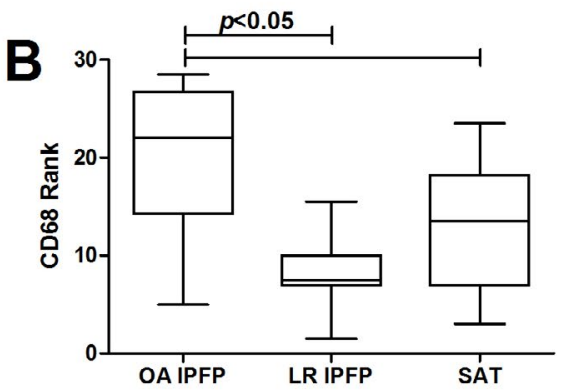
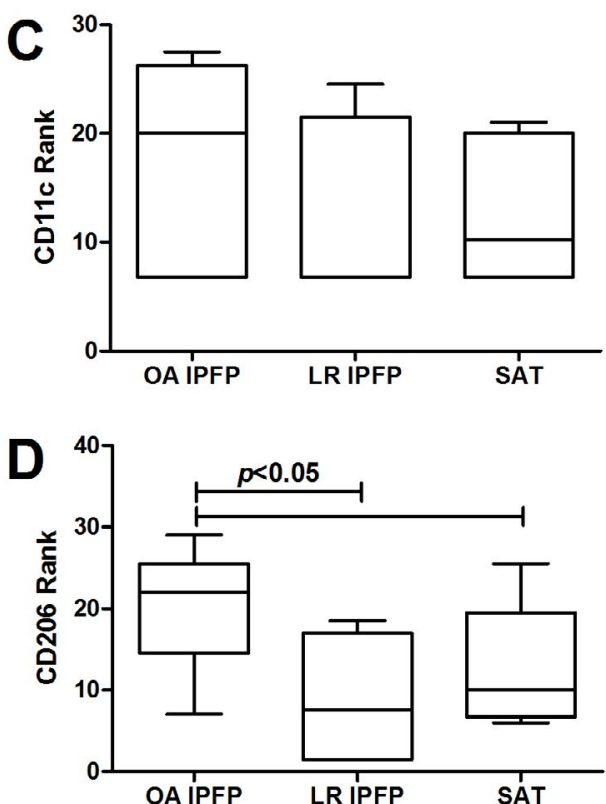

Fig. 5. Macrophages with different phenotypes are present in adipose tissue samples. 13 samples of OA IPFP, 7 samples of LR IPFP and 9 samples of SAT were used. (A) Representative images of immunohistochemcal staining of CD68, CD11c and CD206 positive cells in OA IPFP samples. Positive cells are stained red and indicated with arrows. Ranking of positive staining in adipose tissue samples for (B) CD68, (C) CD11c, and (D) CD206.

\section{Difference between IPFP and SAT in number and phenotype of macrophages}

We hypothesised that the difference in effects of IPFP and SAT on expression of chondrogenic and fibrous tissue genes could be due to the type of macrophages in the adipose tissue. We therefore first characterised the macrophages in the different adipose tissues. IPFP samples from OA donors ranked higher on $\operatorname{CD} 68(p=0.007$ and $p=0.03)$ and CD206 ( $p=0.02$ and $p=0.049)$ positive staining than LR IPFP and SAT samples (Fig. 5B and D). We could not detect differences between obese and lean donors.

\section{Macrophages in IPFP contribute to the effects seen on MSC chondrogenesis}

To investigate whether macrophages present in the IPFP were responsible for the effect seen on MSC chondrogenesis, we added CM from macrophages isolated from IPFP to MSCs during chondrogenesis. IPFP macrophages statistically significantly lowered the COL2A1 and ACAN gene expression (Fig. 6A). Since we applied a very strict selection for CD45+ cells, the remainder fraction still contained a small number of CD45+ cells. This CD45 reduced fraction appeared to be able to inhibit $C O L 2 A 1$ and $A C A N$ gene expression as well (Fig. 6B). To investigate whether this effect was specific for cells isolated from the IPFP, we added CM from human dermal fibroblasts (HDFa) to MSCs during chondrogenesis. CM from HDFa did not influence MSC chondrogenesis (Fig. 6B).

Finally we investigated whether the negative effects of IPFP-derived macrophages on MSC chondrogenesis could be explained by the phenotype of the macrophage. Due to the low number of macrophages we could obtain from IPFP, we used PBMCs that were stimulated to proor anti-inflammatory phenotype. MSCs exposed to CM of $\mathrm{M}(\mathrm{LPS}+\mathrm{IFN \gamma})$ macrophages had lower hyaline cartilage genes expression compared to control and CM of M(IL-4) macrophages (Fig. 6C).

\section{Discussion}

IPFP secretes cytokines, growth factors and adipokines which could influence the joint environment (Clockaerts et al., 2010). In this study, we have shown that the IPFP released factors that inhibit chondrogenic differentiation of MSCs and the production of hyaline cartilage matrix. This 
A
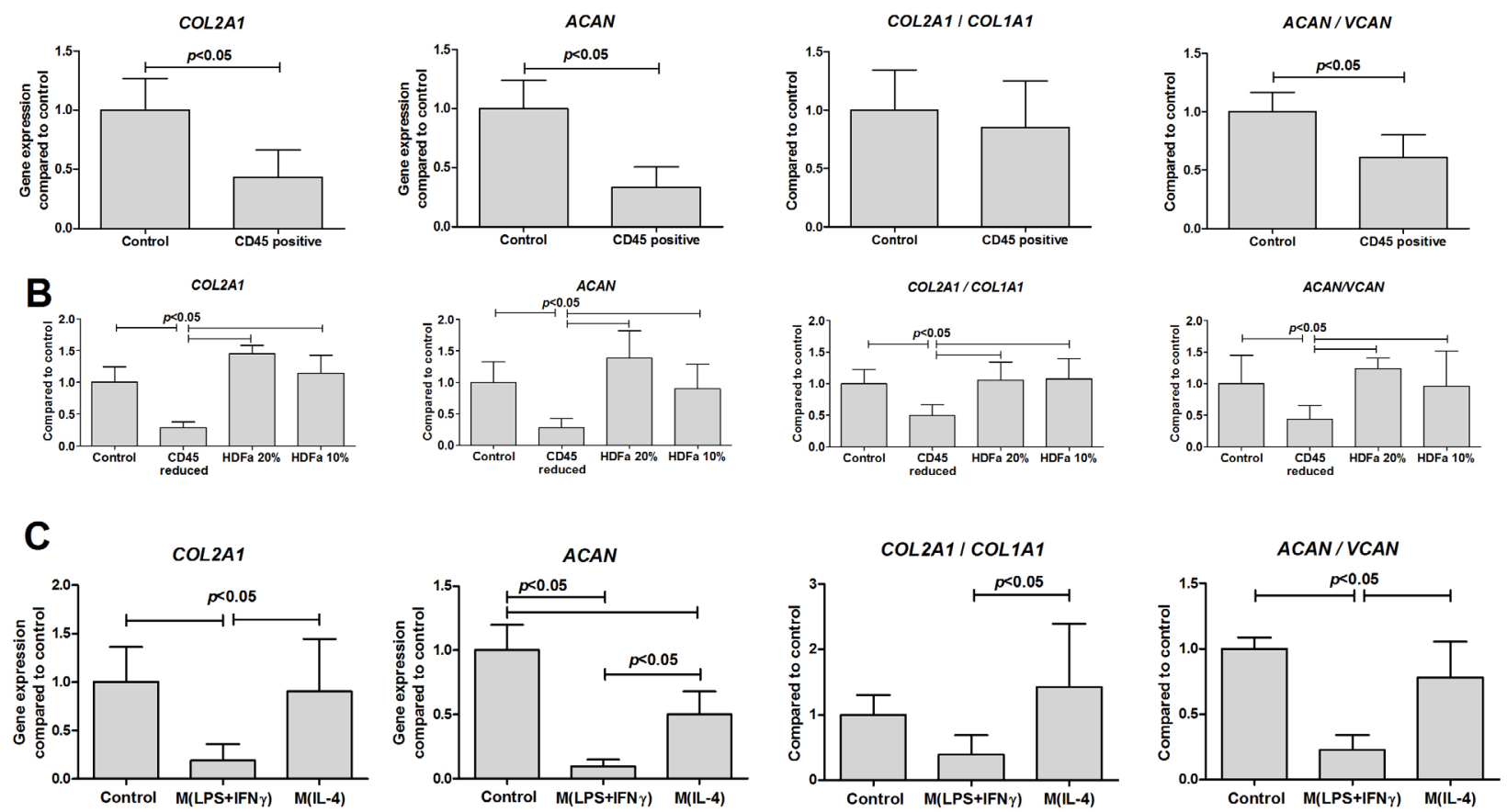

Fig. 6. Macrophages in IPFP contribute to the effects seen on MSC chondrogenesis. MSC alginate beads were treated with CM from day 14-17. The control condition was treated with $25 \%$ unconditioned medium. All conditions were chondrogenically stimulated with TGF $\beta 1$ during the entire culture period. (A) Effect of $50 \%$ CM from CD45+ cells from IPFP on MSC chondrogenesis; (B) Effect $50 \%$ CM from CD45 reduced fraction from IPFP or CM from adult human dermal fibroblasts (HDFa) on MSC chondrogenesis; and (C) Effect of $20 \%$ CM from M(LPS+IFNy) or M(IL-4) macrophages on MSC chondrogenesis. Relative gene expression of COL2A1, ACAN, COL2A1/COL1A1 ratio and ACAN/VCAN ratio in MSCs after treatment. Gene expression was normalised to the control condition per MSC donor. CM were from 6 IPFP donors, $1 \mathrm{HDFa}$ donor or 3 blood donors and used to treat 1 MSC donor. Conditions with CM derived from IPFP were performed in single well and all other conditions were performed in triplicate. Values represent the mean $\pm \mathrm{SD}$.

anti-chondrogenic effect was the same for patients with end stage OA and patients with joint trauma. Furthermore, the IPFP of obese patients did not inhibit chondrogenesis more than IPFP of lean patients. Finally, we have indications that macrophages residing in the IPFP might play an important role in this anti-chondrogenic effect.

Repair tissue formed after marrow stimulation techniques, such as the microfracture procedure, is mainly fibrocartilage, which is of less biomechanical quality than native hyaline cartilage (Furukawa et al., 1980). In the present study, the decrease in COL2A1/COL1A1 and $A C A N / V C A N$ ratios suggests that IPFP not only inhibits chondrogenesis, but also shifts the production from hyaline to fibrocartilage. Fibrocartilage also contains proteoglycans, but instead of aggrecan this is versican. This could be an explanation why we found no reduction in glycosaminoglycan production in alginate beads after IPFP CM treatment, despite a decrease in aggrecan core-protein gene expression. However, this could also be caused by interference of the DMMB assay by alginate especially when low amount of GAG is involved (Enobakhare et al., 1996; Zheng and Levenston, 2015). Analyses on matrix with DMMB and immunohistochemistry can be used more reliably on pellets (Yang et al., 2004). The reduced thionin staining and immunohistochemical staining for collagen type 2 in pellets treated with IPFP CM supports the gene expression results in alginate beads. Our findings indicate that fibrocartilage production by MSCs, taking place after microfracture procedure, could be partly due to factors secreted by the IPFP.

The secretion of factors by IPFP could be influenced by the disease state of the joint. The microfracture procedure and MSC-based treatments are recommended for the treatment of post-traumatic, rather than end-stage OA cartilage defects (Gomoll et al., 2011). Higher levels of inflammatory cytokines were found in post-traumatic than end-stage OA knees (Beekhuizen et al., 2013; Tsuchida et al., 2012). Gandhi et al. (2011) reported that post-traumatic IPFP expresses more pro-inflammatory genes compared to end-stage OA IPFP. We found differences in macrophage number and in particular CD206 positive cells between IPFP from OA and LR knees, albeit factors secreted by both OA and LR IPFP inhibited chondrogenesis. However, we cannot exclude that these effects are due to different cytokines or adipokines.

Another factor that could influence both IPFP and the results of microfracture is obesity (Mithoefer et al., 2005). In obese patients, macrophages in adipose tissue are changed 
towards a chronic inflammatory phenotype with increased production of pro-inflammatory cytokines (Lumeng and Saltiel, 2011). An inflamed obese IPFP would possibly be an explanation for the worse results of microfracture in obese patients. In contrast to this hypothesis, we did not detect an increased anti-chondrogenic effect of IPFP from obese compared to lean donors. To investigate whether the OA process could be of more influence to the factors secreted by IPFP than being obese, experiments with IPFP donors with a low or high BMI but without OA would be required. It is however difficult to obtain sufficient IPFP from non-OA donors.

Independent from joint pathology or obesity, differences have been reported between IPFP and other adipose tissue. IPFP expresses more inflammatory genes, releases more inflammatory factors, and contains more immune cells than SAT (Klein-Wieringa et al., 2011). In our study, the antichondrogenic effect of IPFP was stronger than that of SAT and even after increasing the dosage of SAT CM, the antichondrogenic effect was not increased to the level of IPFP. This suggests that the IPFP releases anti-chondrogenic factors not produced by subcutaneous adipose tissues.

IPFP secretes pro-inflammatory factors, such as TNF- $\alpha$, IL1 $\beta$ and IL6, and adipokines, such as leptin, resistin and adiponectin (Clockaerts et al., 2012). These factors could be produced by the fibroblasts, adipocytes and immune cells residing in the IPFP. A large proportion of the immune cells in the IPFP are macrophages (Bastiaansen-Jenniskens et al., 2012; Klein-Wieringa et al., 2011). Immunohistochemical analysis on IPFP and SAT indicated that IPFP contained more macrophages than SAT. Previously, we have shown that only pro-inflammatory macrophages inhibit chondrogenesis (Fahy et al., 2014) and in the current study, only the pro-inflammatory macrophages shift MSCs to produce fibrocartilage matrix. As a proof of principle, we showed that isolated macrophages from the IPFP were anti-chondrogenic. Although we could not exclude that other cells in the IPFP could also secrete anti-chondrogenic factors, our study does suggest that macrophages in the IPFP play a vital role in inhibiting production of hyaline cartilage and the shift towards fibrocartilage matrix production.

Next to directly negatively affecting chondrogenesis of MSCs, IPFP can influence other joint structures and thereby indirectly influence chondrogenesis. IPFP factors have been shown to stimulate fibrotic processes (BastiaansenJenniskens et al., 2013) and production of pro-inflammatory factors in synovial fibroblasts (Eymard et al., 2014). In this way, synovium can then inhibit chondrogenesis (Fahy et $a l ., 2014)$. In contrast to the effect on synovium, the IPFP inhibits catabolic mediators in cartilage explants stimulated with or without IL1 $\beta$ (Bastiaansen-Jenniskens et al., 2012). This shows that different tissues in the joint can react differently to factors secreted by the IPFP and the interplay between all joint tissues will determine the eventual effect on cartilage repair. The use of co-cultures of several joint tissues would be required to elucidate this in future studies and to show the potential effect of modulation.

In summary, we have shown that the IPFP is a source of factors that inhibit chondrogenic differentiation of MSCs and shift the production from hyaline to fibrocartilage matrix. This anti-chondrogenic effect of adipose tissue was specific for IPFP and not subcutaneous adipose tissue. Furthermore, this anti-chondrogenic effect was not more in obese donors than lean donors and was not different between donors with post-joint trauma or end-stage OA. Finally, the IPFP and specifically the macrophages in the tissue could therefore be targets for future therapies to improve the joint environment for MSC chondrogenesis and thereby improve the outcome of the microfracture procedure and future MSC-based treatments for the repair of cartilage defects.

\section{Acknowledgements}

We would like to thank Pieter Koen Bos for the very helpful discussions and critical reading of the manuscript, and Johannes Lehmann for his assistance on setting up the MACS and FACS. This study was funded by the European Union's 7th Framework Programme under agreement no. NMP3-SL-2010-24599 and the Dutch Arthritis Association, and performed within the Postgraduate School Molecular Medicine, Erasmus MC University Medical Center, The Netherlands. This research was also facilitated by a National University of Ireland travelling grant. Y. Bastiaansen-Jenniskens is supported by a Veni grant from the Dutch Technology foundation STW. WW, RR, YBJ and GVO designed the study; WW, RR and NF acquired the data; WW, RR, NF, JV, SC, YBJ and GVO analysed and interpreted the data; WW, YBJ and GVO drafted the manuscript; all authors revised the manuscript critically and approved the final version. We wish to confirm that there are no known conflicts of interest associated with this publication and there has been no significant financial support for this work that could have influenced its outcome.

\section{References}

Bastiaansen-Jenniskens YM, Clockaerts S, Feijt C, Zuurmond AM, Stojanovic-Susulic V, Bridts C, de Clerck L, DeGroot J, Verhaar JA, Kloppenburg M, van Osch GJ (2012) Infrapatellar fat pad of patients with end-stage osteoarthritis inhibits catabolic mediators in cartilage. Ann Rheum Dis 71: 288-294.

Bastiaansen-Jenniskens YM, Wei W, Feijt C, Waarsing JH, Verhaar JA, Zuurmond AM, Hanemaaijer R, Stoop R, van Osch GJ (2013) Stimulation of fibrotic processes by the infrapatellar fat pad in cultured synoviocytes from patients with osteoarthritis: a possible role for prostaglandin f2alpha. Arthritis Rheum 65: 2070-2080.

Beekhuizen M, Gierman LM, van Spil WE, Van Osch GJ, Huizinga TW, Saris DB, Creemers LB, Zuurmond AM (2013) An explorative study comparing levels of soluble mediators in control and osteoarthritic synovial fluid. Osteoarthritis Cartilage 21: 918-922.

Clockaerts S, Bastiaansen-Jenniskens YM, Runhaar J, Van Osch GJ, Van Offel JF, Verhaar JA, De Clerck 
LS, Somville J (2010) The infrapatellar fat pad should be considered as an active osteoarthritic joint tissue: a narrative review. Osteoarthritis Cartilage 18: 876-882.

Clockaerts S, Bastiaansen-Jenniskens YM, Feijt C, De Clerck L, Verhaar JA, Zuurmond AM, StojanovicSusulic V, Somville J, Kloppenburg M, van Osch GJ (2012) Cytokine production by infrapatellar fat pad can be stimulated by interleukin 1beta and inhibited by peroxisome proliferator activated receptor alpha agonist. Ann Rheum Dis 71: 1012-1018.

EnobakhareBO, BaderDL, LeeDA(1996)Quantification of sulfated glycosaminoglycans in chondrocyte/alginate cultures, by use of 1,9-dimethylmethylene blue. Anal Biochem 243: 189-191.

Eymard F, Pigenet A, Citadelle D, Flouzat-Lachaniette $\mathrm{CH}$, Poignard A, Benelli C, Berenbaum F, Chevalier X, Houard X (2014) Induction of an inflammatory and prodegradative phenotype in autologous fibroblast-like synoviocytes by the infrapatellar fat pad from patients with knee osteoarthritis. Arthritis Rheumatol 66: 2165-2174.

Fahy N, de Vries-van Melle ML, Lehmann J, Wei W, Grotenhuis N, Farrell E, van der Kraan PM, Murphy JM, Bastiaansen-Jenniskens YM, van Osch GJ (2014) Human osteoarthritic synovium impacts chondrogenic differentiation of mesenchymal stem cells via macrophage polarisation state. Osteoarthritis Cartilage 22: 1167-1175.

Frisbie DD, Oxford JT, Southwood L, Trotter GW, Rodkey WG, Steadman JR, Goodnight JL, McIlwraith CW (2003) Early events in cartilage repair after subchondral bone microfracture. Clin Orthop Relat Res: 215-227.

Furukawa T, Eyre DR, Koide S, Glimcher MJ (1980) Biochemical studies on repair cartilage resurfacing experimental defects in the rabbit knee. J Bone Joint Surg Am 62: 79-89.

Gandhi R, Takahashi M, Virtanen C, Syed K, Davey JR, Mahomed NN (2011) Microarray analysis of the infrapatellar fat pad in knee osteoarthritis: relationship with joint inflammation. J Rheumatol 38: 1966-1972.

Gierman LM, Wopereis S, van El B, Verheij ER, Werff-van der Vat BJ, Bastiaansen-Jenniskens YM, van Osch GJ, Kloppenburg M, Stojanovic-Susulic V, Huizinga TW, Zuurmond AM (2013) Metabolic profiling reveals differences in concentrations of oxylipins and fatty acids secreted by the infrapatellar fat pad of donors with endstage osteoarthritis and normal donors. Arthritis Rheum 65: $2606-2614$.

Gomoll AH, Farr J, Gillogly SD, Kercher JS, Minas T (2011) Surgical management of articular cartilage defects of the knee. Instr Course Lect 60: 461-483.

Grant RW, Dixit VD (2015) Adipose tissue as an immunological organ. Obesity (Silver Spring) 23: 512-518.

Grotenhuis N, Vd Toom HF, Kops N, Bayon Y, Deerenberg EB, Mulder IM, van Osch GJ, Lange JF, Bastiaansen-Jenniskens YM (2014) In vitro model to study the biomaterial-dependent reaction of macrophages in an inflammatory environment. Br J Surg 101: 983-992.

Heldens GT, Blaney Davidson EN, Vitters EL, Schreurs BW, Piek E, van den Berg WB, van der Kraan PM (2012) Catabolic factors and osteoarthritis-conditioned medium inhibit chondrogenesis of human mesenchymal stem cells. Tissue Eng Part A 18: 45-54.
Hunziker EB, Lippuner K, Keel MJ, Shintani N (2015) An educational review of cartilage repair: precepts \& practice - myths \& misconceptions - progress \& prospects. Osteoarthritis Cartilage 23: 334-350.

Irie K, Uchiyama E, Iwaso H (2003) Intraarticular inflammatory cytokines in acute anterior cruciate ligament injured knee. Knee 10: 93-96.

Kaul G, Cucchiarini M, Remberger K, Kohn D, Madry H (2012) Failed cartilage repair for early osteoarthritis defects: a biochemical, histological and immunohistochemical analysis of the repair tissue after treatment with marrow-stimulation techniques. Knee Surg Sports Traumatol Arthrosc 20: 2315-2324.

Klein-Wieringa IR, Kloppenburg M, BastiaansenJenniskens YM, Yusuf E, Kwekkeboom JC, El-Bannoudi $\mathrm{H}$, Nelissen RG, Zuurmond A, Stojanovic-Susulic V, Van Osch GJ, Toes RE, Ioan-Facsinay A (2011) The infrapatellar fat pad of patients with osteoarthritis has an inflammatory phenotype. Ann Rheum Dis 70: 851-857.

Lumeng CN, Saltiel AR (2011) Inflammatory links between obesity and metabolic disease. J Clin Invest 121: 2111-2117.

Mithoefer K, Williams RJ, 3rd, Warren RF, Potter HG, Spock CR, Jones EC, Wickiewicz TL, Marx RG (2005) The microfracture technique for the treatment of articular cartilage lesions in the knee. A prospective cohort study. J Bone Joint Surg Am 87: 1911-1920.

Mosser DM, Edwards JP (2008) Exploring the full spectrum of macrophage activation. Nat Rev Immunol 8: 958-969.

Murray PJ, Allen JE, Biswas SK, Fisher EA, Gilroy DW, Goerdt S, Gordon S, Hamilton JA, Ivashkiv LB, Lawrence T, Locati M, Mantovani A, Martinez FO, Mege JL, Mosser DM, Natoli G, Saeij JP, Schultze JL, Shirey KA, Sica A, Suttles J, Udalova I, van Ginderachter JA, Vogel SN, Wynn TA (2014) Macrophage activation and polarization: nomenclature and experimental guidelines. Immunity 41: 14-20.

Shapiro F, Koide S, Glimcher MJ (1993) Cell origin and differentiation in the repair of full-thickness defects of articular cartilage. J Bone Joint Surg Am 75: 532-553.

Steadman JR, Miller BS, Karas SG, Schlegel TF, Briggs KK, Hawkins RJ (2003) The microfracture technique in the treatment of full-thickness chondral lesions of the knee in National Football League players. J Knee Surg 16: 83-86.

Tsuchida AI, Beekhuizen M, Rutgers M, van Osch GJ, Bekkers JE, Bot AG, Geurts B, Dhert WJ, Saris DB, Creemers LB (2012) Interleukin-6 is elevated in synovial fluid of patients with focal cartilage defects and stimulates cartilage matrix production in an in vitro regeneration model. Arthritis Res Ther 14: R262.

Wehling N, Palmer GD, Pilapil C, Liu F, Wells JW, Muller PE, Evans CH, Porter RM (2009) Interleukin-1 beta and tumor necrosis factor alpha inhibit chondrogenesis by human mesenchymal stem cells through NF-kappaBdependent pathways. Arthritis Rheum 60: 801-812.

Yang IH, Kim SH, Kim YH, Sun HJ, Kim SJ, Lee JW (2004) Comparison of phenotypic characterization between "alginate bead" and "pellet" culture systems as chondrogenic differentiation models for human mesenchymal stem cells. Yonsei Med J 45: 891-900. 
Zheng CH, Levenston ME (2015) Fact versus artifact: avoiding erroneous estimates of sulfated glycosaminoglycan content using the dimethylmethylene blue colorimetric assay for tissue-engineered constructs. Eur Cell Mater 29: 224-236.

\section{Discussion with Reviewers}

Reviewer I: This study presents the intriguing interpretation that the infrapatellar fat pad but not subcutaneous adipose contains macrophages that impede chondrogenic differentiation of mesenchymal stromal/stem cells through a paracrine mechanism. Much of the interpretation hinges on $\mathrm{qPCR}$ data (presented in four of the six figures). Yet, quantitative aspects of the qPCR results are unclear, because values have been arbitrarily set to 1 for one of the samples. Before one can define ratios, it is important to establish that values of two parameters are sufficiently robust.

Authors: We understand that setting the condition which was treated with control medium to 1.0 can create concerns. However, since each MSC donor has a different chondrogenic potential, setting the "control" condition with unconditioned medium to 1.0 for each donor allows better visualisation of the effect of conditioned medium treatment. To be sure that our qPCR data are sufficiently robust, we have analysed the efficiency of the primers and confirmed stable expression of the two housekeeping genes used.

Reviewer II: Presuming that future studies confirm that activated, pro-inflammatory macrophages are the primary source of anti-chondrogenic factors secreted by the IPFP, how might this population be targeted to enhance MSC-based cartilage repair? For example, assuming that these cells could be specifically targeted in vivo, what might be the negative consequences of ablating them altogether? Alternatively, might it be possible to shift this subpopulation to an anti-inflammatory phenotype in situ? Authors: Our study suggests that pro-inflammatory macrophages could be a target in the future to improve MSC-based cartilage repair. According to our work, antiinflammatory macrophages are not anti-chondrogenic and removing all macrophages could increase inflammation by other inflammatory cells such as T-cells. However, it has been proven possible to shift macrophage phenotype from a pro-inflammatory to an anti-inflammatory phenotype using drugs (Van der Garde et al., 2014; Zhang et al., 2015). It would be interesting to investigate whether the use of these drugs can change the macrophage phenotype in the IPFP and therefore reduce the anti-chondrogenic effect of the IPFP. If delivered locally into the IPFP, this might provide a way to improve the joint environment before performing MSC-based cartilage repair procedures.

Reviewer II: Given the recent identification of a chronic inflammatory phenotype within the adipose tissues of obese patients, it was somewhat surprising IPFP from donors with high BMI did not demonstrate altered anti-chondrogenic effects compared to those with lower BMI. As you point out, it would be interesting to make this comparison after eliminating the confounding factor of pre-existing joint disease. If patient enrolment were not a limiting factor, what clinical population and treatment effects would you include in future studies of IPFP anti-chondrogenic activity? For example, are there acute dietary changes for patients with high BMI that have been shown to lower the inflammatory phenotype within their adipose tissues? Authors: Metabolic dysregulation rather than an increased BMI alone has been shown to be a risk factor for the development of OA (Zhuo et al., 2012). It would be interesting to investigate whether IPFP from donors with metabolic syndrome would be more anti-chondrogenic than from donors without metabolic syndrome. In this way, we could maybe better identify the patients in most need of joint modulation prior to cartilage repair procedures. It has also been shown that an increased intake of omega-3 fatty acids could decrease chronic inflammation (Simopoulus, 2002). Furthermore, it has been shown in mice that dietary fatty acid content could regulate wound healing and OA severity following joint injury (Wu et al., 2015). It would therefore be interesting to investigate whether the IPFP from donors, without OA, who had increased intake of omega- 3 fatty acids are less anti-chondrogenic compared to those on a 'regular' diet. In this way, in the future, we can investigate whether dietary changes would be a possible strategy to modulate IPFP inflammation and improve cartilage repair procedures.

\section{Additional References}

Simopoulos AP (2002) Omega-3 fatty acids in inflammation and autoimmune diseases. J Am Coll Nutr 21: 495-505.

van de Garde MD, Martinez FO, Melgert BN, Hylkema MN, Jonkers RE, Hamann J (2014) Chronic exposure to glucocorticoids shapes gene expression and modulates innate and adaptive activation pathways in macrophages with distinct changes in leukocyte attraction. J Immunol 192: 1196-1208.

Wu CL, Jain D, McNeill JN, Little D, Anderson JA, Huebner JL, Kraus VB, Rodriguiz RM, Wetsel WC, Guilak F (2015) Dietary fatty acid content regulates wound repair and the pathogenesis of osteoarthritis following joint injury. Ann Rheum Dis 74: 2076-2083.

Zhang O, Zhang J (2015) Atorvastatin promotes human monocyte differentiation toward alternative M2 macrophages through p38 mitogen-activated protein kinase-dependent peroxisome proliferator-activated receptor gamma activation. Int Immunopharmacol 26: 58-64.

Zhuo Q, Yang W, Chen J, Wang Y (2012) Metabolic syndrome meets osteoarthritis. Nat Rev Rheumatol 8: 729-737.

Editor's Note: Scientific Editor in charge of the paper: Chris Evans. 